\title{
Retention of vinyl chloride in the human lung
}

\author{
J KRAJEWSKI, M DOBECKI, AND J GROMIEC \\ From the Department of Chemical Air Pollutants of the Institute of Occupational Medicine, Lodz, \\ Poland
}

ABSTRACT Experiments with volunteers showed that $42 \%$ of an inhaled dose of vinyl chloride is retained in the lungs. This value is independent of the concentration of vinyl chloride in the air. Elimination of vinyl chloride through the lungs is negligible since its concentration in expired air decreases immediately after the cessation of exposure.

Interest in the toxic action of vinyl chloride (VC) has grown recently following the increased incidence of hepatic angiosarcoma found among VC polymerisation workers.

In industry the main route of $\mathrm{VC}$ absorption is through the lung. No data on skin absorption in man are available, but it cannot be excluded since the compound was found to penetrate the skin of exposed monkeys. ${ }^{1}$ The retention rate of $\mathrm{VC}$ in the lung was reported by Buchter et $a l^{2}$ to be $26-28 \%$, but these results were obtained from only two individuals exposed to $2.5 \mathrm{ppm} \mathrm{VC}$ and may thus be subject to error. The purpose of the present study was to determine the percentage retention of $\mathrm{VC}$ in the lung in man.

\section{Method}

Five healthy male volunteers aged from 26 to 31 were carefully examined by a doctor before and after exposure, and medical supervision was provided for each experiment. The volunteers were exposed to four atmospheric concentrations of $\mathrm{VC}$ with a $1 \mathrm{~m}^{3}$ toxicological chamber serving as the source of VCair mixtures. VC monomer from a pressure cylinder was mixed with precleaned air in a two-step flowdilution system to give the final concentrations. Volunteers were exposed for six hours to VC concentrations $60 \mathrm{mg} / \mathrm{cm}^{3}(24 \mathrm{ppm}), 30 \mathrm{mg} / \mathrm{cm}^{3}(12$ $\mathrm{ppm}), 15 \mathrm{mg} / \mathrm{cm}^{3}(6 \mathrm{ppm})$, and $7.5 \mathrm{mg} / \mathrm{cm}^{3}(3 \mathrm{ppm})$; values equal to $2,1,0.5$, and 0.25 of the Polish maximum allowable concentration respectively. The subject remained outside the chamber and inhaled the air from inside through a gas mask connected with the interior of the chamber.

Received 29 October 1979

Accepted 19 November 1979
Samples of inhaled air were collected into $500 \mathrm{~cm}^{3}$ gas pipettes every five minutes, the expired air was sampled using charcoal tubes, and four to five 10minute samples were taken every hour. The VC concentration in both the inhaled and the expired air was determined by gas chromatography ${ }^{3}$; determinations were possible down to $0.3 \mathrm{mg} / \mathrm{m}^{3}$. The difference between the concentration in the inhaled and expired air was calculated to obtain the percentage retention of VC in the lung. Samples of exhaled air were also collected for the first 90 minutes after the cessation of exposure to determine the rate of elimination of $\mathrm{VC}$ from the lung.

\section{Results and discussion}

The percentage retention of $\mathrm{VC}$ in the lung is shown in the figure. The value was independent of the VC concentration, averaging $42 \%$ and reaching the highest level of $46 \%$ in the first 15 minutes of exposure. In all the experiments the retention rate

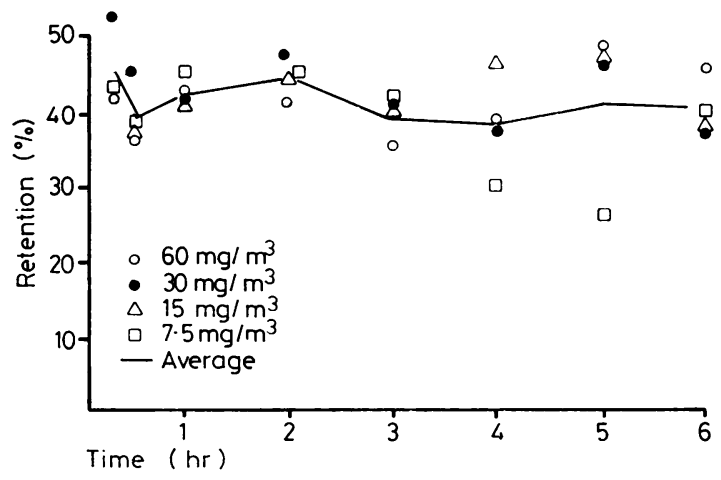

Percentage retention of vinyl chloride in the lung. 
